\title{
TEM Observation of Deformation under Nanoindentation in Single Crystal Ni- based Superalloys
}

\author{
A.S. Khali1 ${ }^{1}$, G. Fuchs ${ }^{2}$, J. Turner ${ }^{3}$, B. $\mathrm{Ozdol}^{3}$, C. Song ${ }^{3}$, S. Vachhani ${ }^{4}$ and A.M. Minor ${ }^{3,5}$ \\ 1. Metallurgy and Mining Department, Tabbin Institute for Metallurgical Studies, Cairo, Egypt. \\ 2. Department of Material Sciences and Engineering, University of Florida, Gainesville, USA. \\ ${ }^{3 .}$ National Center for Electron Microscopy, Molecular Foundry, Lawrence Berkeley National \\ Laboratory, Berkeley, USA. \\ 4. Bruker, Inc., Minneapolis, USA. \\ 5. Department of Material Sciences and Engineering, University of California, Berkeley, USA.
}

Single crystal Ni-based superalloys are developed for applications involving high mechanical stresses at elevated temperatures. They have been exclusively used over the past three decades for turbine blades in jet engines and recently in land-based gas turbines for power generation. These superalloys are alloyed with different refractory elements to further improve their high temperature mechanical capability. Among more than ten alloying elements usually added to superalloys, the addition of $\mathrm{Re}$ and Ru play a prominent role in promoting their mechanical capability at higher temperatures [1].

In this study, samples were sectioned from two Ni-based superalloy single crystal bars $(1.27 \mathrm{~cm}$ in diameter) of $<001>$ orientation prepared by the Bridgman method. Superalloy (A) has the following composition in wt\% $(4.1 \% \mathrm{Cr}, 12.2 \% \mathrm{Co}, 5.85 \% \mathrm{~W}, 8.6 \% \mathrm{Ta}, 5.5 \% \mathrm{Al}, 0.1 \% \mathrm{Hf}$ and Ni \% Bal. $)$ while superalloy (B) includes 6\% Re and 3\% Ru (in wt $\%$ ). The samples were heat treated in an inert atmosphere through the standard double-aging heat treatment for Ni-based single crystal superalloys. The samples were mechanically polished using common metallographic protocols and finished by vibration-polishing in $50 \mathrm{~nm}$ alumina slurry for 48 hours. A Hysitron TI-950 nanoindenter was used to introduce an array of 6x6 indents for each sample by a Berkovich type indenter at load of $12 \mathrm{mN}$. TEM foils across the center of indents were prepared by using an FEI 235 Strata focused ion beam system. The subsequent TEM observations were made on a Philips CM-200 FEG operating at $200 \mathrm{kV}$ in a scanning-transmission mode.

The deformation areas due to the indents in both the (001) Ni-based single crystals (A) and (B) are shown in figure 1(a) and 1(b). The indent and the underlying deformation in superalloy (A) depicted in figure 1(a) extends deeper than in superalloy (B) shown in figure 1(b). Moreover, superalloy (A) exhibits larger intermetallic $\gamma^{\prime}$ phase cube-like precipitates relative to these in superalloy (B). The measured higher value of nanohardness in superalloy (B) further supports our observation. The effect of adding Re is to strengthen superalloy (B) through solid solution strengthening of the metallic $\gamma$ phase and to raise its melting temperature [2]. This is achieved on microstructural level by increasing the misfit strain between the $\gamma^{\prime}$ precipitates and the $\gamma$ matrix as Re increases the lattice constant of $\gamma$ phase. Furthermore, Re also retards the coarsening of the diffusion-controlled process of the precipitates at higher temperatures [2] which might explain the smaller $\gamma^{\prime}$ phase observed in superalloy (B). The addition of $\mathrm{Ru}$ provides excellent microstructure stability that allows for the benefits gained from Re and it permits Re to distribute more homogenously between the $\gamma$ and $\gamma^{\prime}$ phases. Ru also strengthens the superalloy (B) through solid solution strengthening of the $\gamma^{\prime}$ phase [3]. The synergistic additions of Re and $\mathrm{Ru}$ together also improves the observed microstructure by obtaining better aligned, more cubicshaped $\gamma^{\prime}$ particles, with smaller size and larger misfits. These factors might help to increase superalloy (B) performance at higher temperatures and allow it to endure higher mechanical stress [4-7]. 


\section{References:}

[1] Roger C. Reed, in "The Superalloys Fundamentals and Applications" (Cambridge University Press, Cambridge) 2006.

[2] K. Durst and M. Göken, Materials Science and Engineering A, 387-389 (2004), p. 312.

[3] S. Neumeier, F. Pyczak and M. Göken, Philosophical Magazine 91 (2011), p. 4187.

[4] Tersa M. Pollock and Sammy Tin, Journal of Propulsion and Power V22, no.2 (2006), p. 361.

[5] A.S. Khalil is greatly indebted to his father, the late Prof. Saied E. Khalil (1938-2017), Emeritus Professor of Physical Metallurgy at Tabbin Institute for Metallurgical Studies for his profound support during this work.

[6] A.S. Khalil acknowledges Dr. Jacqueline B. Wahl, Cannon-Muskegon Corporation for kindly providing CMSX-4 superalloy as a reference. And Prof. Michael Mills, McDougal Professor of Material Sciences and Engineering at The Ohio State University for his useful discussions.

[7] This work (project no. 3590) was performed at the Molecular Foundry, Lawrence Berkeley National Laboratory and was supported by the DOE Office of Science under Contract No. DE-AC02$05 \mathrm{CH} 11231$.
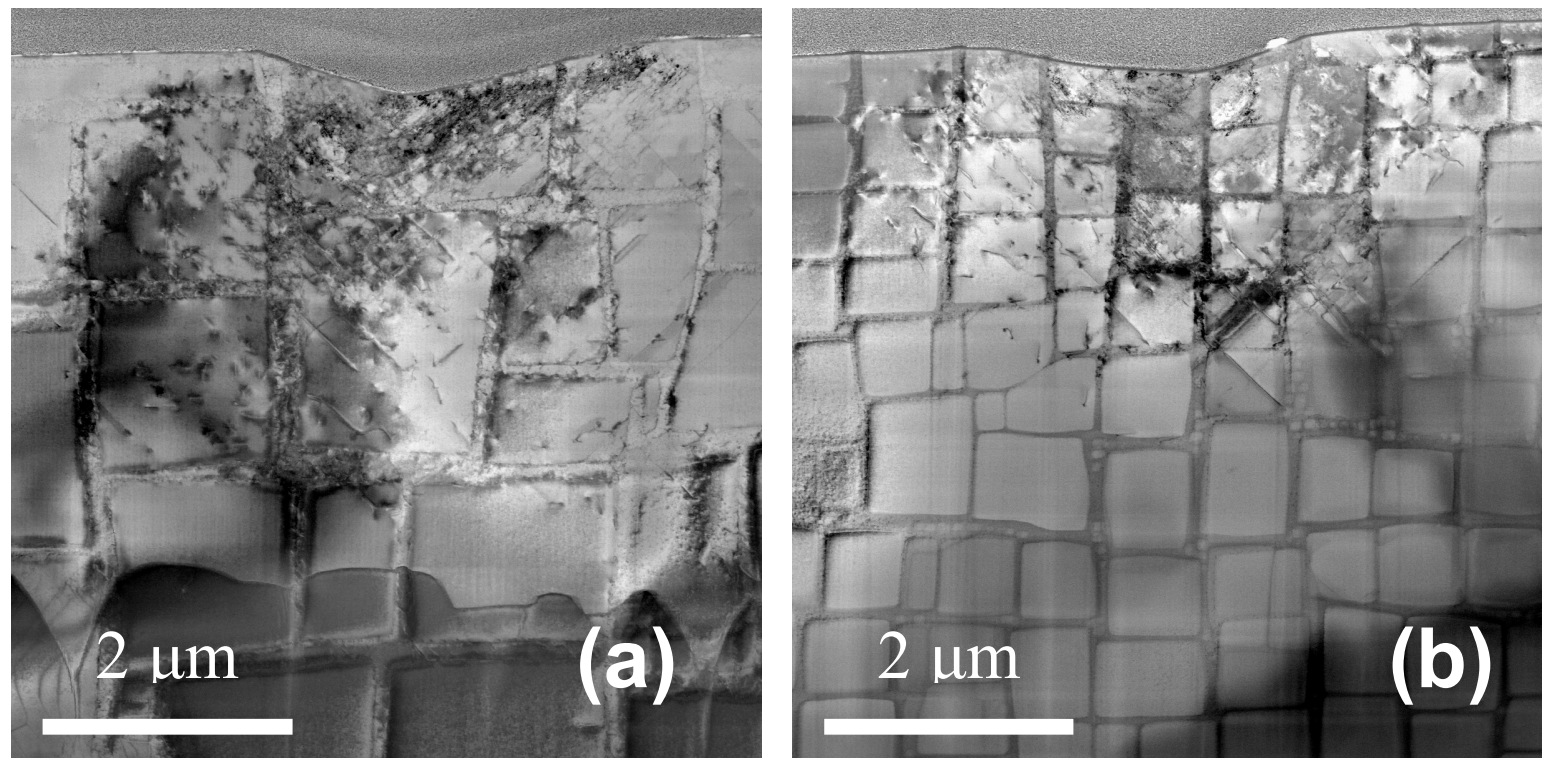

Figure 1. Micrographs of cross section of indents resulting from a $12 \mathrm{mN}$ load, shown in (a) an indent in superalloy (A) with $0 \% \mathrm{Re}$ and $0 \% \mathrm{Ru}$, and in (b) an indent in superalloy (B) which includes $6 \% \mathrm{Re}$ and $3 \% \mathrm{Ru}($ in $\mathrm{wt} \%)$. 